\title{
Biografische Dynamiken moralischer Positionierungen in Prozessen sozialer Reintegration
}

\section{Eine Längsschnittanalyse im Kontext von Drogenkonsum, Kriminalität und Arbeit}

\section{Franz Zahradnik}

Journal für Psychologie, 28(2), 78-100

https://doi.org/10.30820/0942-2285-2020-2-78

CC BY-NC-ND 3.0 DE

www.journal-fuer-psychologie.de

\section{Zusammenfassung}

Sich von anderen in moralischer Weise abzugrenzen ist eine verbreitete Reaktionsweise, wenn man sich durch äußere Zuschreibungen in die Nähe von stigmatisierten Gruppen wie etwa Drogenkonsument*innen gestellt sieht. Dieser bekannte, bisher nur im Querschnitt untersuchte Forschungsbefund wird im vorliegenden Beitrag anhand einer theorieorientierten Fallrekonstruktion von vier Längsschnittinterviews mit einem wegen Drogendelikten strafrechtlich verurteilten Mann tiefergehend untersucht. Es zeigt sich im Zeitverlauf, dass sich verändernde soziale Kontexte je spezifische moralisch begründete Abgrenzungen und $\mathrm{Zu}$ gehörigkeitsbekundungen mithervorbringen. Als moralische Differenzierungslinien dienen vor allem Arbeitsorientierung, alltägliche Lebensführung, Kontrolle des Drogenkonsums sowie Kriminalität. Die narrativen Positionierungen verweisen auf Anerkennungs- und Statuskonflikte, die in den verschiedenen Phasen auf spezifische Weise herausgefordert werden und sich teils deutlich zuspitzen. Es zeigt sich, dass hierbei die jeweiligen Erfahrungen der Einbindung in Institutionen der Hilfe und Kontrolle eine bedeutsame Rolle spielen. Die Ablösung von diesen Institutionen, insbesondere durch die Integration in Erwerbsarbeit, beruhigt die Abgrenzung von stigmatisierten Gruppen und bringt neue moralische Differenzierungslinien hervor.

Schlüsselwörter: Qualitativer Längsschnitt, Reintegration, Drogen, Kriminalität, Arbeit, Narrationsanalyse, moralische Positionierung 


\section{Summary}

Biographical dynamics of moral positionings in processes of social reintegration. A longitudinal analysis in the context of drug use, criminality and work Morally distancing oneself from others is a common reaction when being allocated in the vicinity of stigmatized groups such as drug users by external attributions. The present article will examine this well-known research finding, which has so far only been examined in a cross-sectional manner, in greater depth on the basis of a theory-oriented case reconstruction of four longitudinal interviews with a man convicted of drug offences. In the course of time, changing social contexts generate specific morally based narrative positionings of dissociation and association. The main lines of moral differentiation are work orientation, everyday life, control of drug use, and criminality. The narrative positionings refer to conflicts of recognition and status that are challenged in specific ways in the various phases and in some cases come to a head. It appears that the particular experiences of involvement in institutions of help and control play a significant role here. Detachment from these institutions, especially through integration into employment, settles the narrative dissociation from stigmatized groups and produces new lines of moral differentiation.

Keywords: Qualitative longitudinal study, reintegration, drugs, crime, work, narrative analysis, moral positioning

\section{Einleitung}

Moralische Kategorisierungen, die Personen und Gruppen nach bestimmten Merkmalen auf dem Kontinuum von besser und schlechter verorten, prägen die Gesellschaft auf allen Ebenen (Hirschauer 2014 und 2017). Vor allem Kategorien sozialer Probleme, wie etwa Drogenkonsum, werden im öffentlichen Diskurs vor dem Hintergrund kultureller Wissensbestände und Wertstrukturen in je spezifischer Weise geformt und schließlich in Organisationen der Problembearbeitung als Maßnahmen und Programme institutionalisiert. Diese Kategorisierungsprozesse sind grundlegend durch moralische Bewertungen bestimmt, die wiederum »Grundlage und Bezugspunkt für das Selbstbild der Betroffenen « (Groenemeyer 2010, 18) sind. So wird der Konsum bestimmter Drogen wie etwa Heroin im öffentlichen Diskurs häufig mit Kontrollverlust und Willensschwäche verbunden, was sich zum Beispiel in der weitverbreiteten Negativklassifizierung als Junkie äußert. Drogenkonsum ist zwar mittlerweile weniger Gegenstand von Kriminalisierung, aber auch die pathologisierende Betrachtung und institutionelle Behandlung von Sucht-als-Krankheit weist ein deutliches Stigmatisierungspotenzial auf (SchmidtSemisch 2010). Personen, die durch moralische (Ab-)Werturteile angerufen werden, müssen auf verschiedenste Arten versuchen, mit diesen Zuschreibungen umzugehen. 
Eine weitverbreitete Reaktionsweise besteht in der moralischen Distanzierung von Personen oder Gruppen, in deren Nähe man sich gestellt sieht, die man aber weiter unten in der sozialen Rangordnung verortet, um sich seiner selbst zu vergewissern (Neckel 1991). Drogenkonsument*innen grenzen sich in ihren Erzählungen deshalb häufig von denjenigen ab, denen sie zuschreiben, ihren Konsum nicht kontrollieren zu können (Copes 2016).

Im vorliegenden Beitrag werden die moralischen Positionierungen aus vier Längsschnittinterviews mit einem Mann rekonstruiert, der nach langjährigem Drogenkonsum und -handel zu einer Haftstrafe verurteilt wurde und ersatzweise an einer Suchttherapie teilgenommen hat. Mittels der etwa alle eineinhalb Jahre geführten Interviews kann so ausgehend von der Endphase der Drogentherapie über einen Zeitraum von circa fünf Jahren hinweg analysiert werden, auf welche Weise sich moralische Zugehörigkeitsbekundungen sowie Abgrenzungen im Zeitverlauf verändern und wie dies an soziale Kontexte rückgebunden wird. Im Zentrum steht die Frage, auf welche Weise moralische Positionierungen als Ausdruck und Verarbeitungsweise von Anerkennungs- und Statuskonflikten gelten können. Durch solch eine längsschnittliche Einzelfallanalyse können die teils parallel, teils ungleichzeitig oder gegenläufig verlaufenden Dynamiken moralischer Positionierungen erschlossen werden.

\section{Genese und Dynamiken moralischer Kategorisierungen}

Personen, die aufgrund drogenbezogener Delikte strafrechtlich verurteilt wurden, werden auf unterschiedlichen gesellschaftlichen Ebenen mit moralischen Vorurteilen konfrontiert und kommen zumeist mit verschiedenen Institutionen der Hilfe und Kontrolle wie etwa therapeutischen Einrichtungen, Bewährungsdiensten sowie der Arbeits- und Sozialverwaltung in Kontakt. Diese Institutionen haben sich historisch in je spezifischen Prozessen öffentlicher und politischer Problematisierung herausgebildet und operieren auf der Grundlage bestimmter gesellschaftlich und politisch akzeptierter Definitionen von Kategorien sozialer Probleme. Im Zuge der Transformation von Personen in den Stand eine*r Klient*in verändert sich durch die wertende Problemzuschreibung immer auch ihr moralischer Status. Den Klient*innen werden im institutionellen Kategorisierungsprozess jeweils in spezifischer Weise Schuld und Verantwortung zugeschrieben, was sich zum Beispiel in der Behandlung als Täter*in oder Opfer beziehungsweise würdige* oder unwürdige*r Anspruchsteller*in äußert. Aber auch die Klient*innen haben die Möglichkeit, sich entsprechend den institutionellen Erwartungen zu präsentieren und dadurch gegebenenfalls eine bessere Behandlung zu erfahren (Groenemeyer 2010). Diese Prozesse werden nun bezogen auf die institutionellen Kontexte Drogenkonsum, Kriminalität und Arbeit kurz skizziert, wobei sich stellenweise Überschneidungen ergeben. 
In Bezug auf Drogenkonsum hat in den letzten Jahrzehnten eine Verschiebung von kriminalisierenden hin zu pathologisierenden Deutungsweisen stattgefunden und sich in besonderem Maße die Konstruktion von Sucht-als-Krankheit verfestigt (Reinarman 2005). In diesem Prozess wurde die Definitionsmacht der medizinischen Profession hinsichtlich der Entscheidung über die Zuweisung in eine Therapieeinrichtung statt der Unterbringung in einer Haftanstalt deutlich erweitert. Ihre Vertreter*innen bestimmen maßgeblich, ob die betreffende Person der Problemkategorie entspricht, also zum Beispiel als suchtkrank und behandlungswillig gelten kann. Gegenüber den Vertreter*innen der Justiz muss von der/dem Klientin/Klienten gleichzeitig aber auch glaubhaft signalisiert werden, dass strafrechtlich relevante Verhaltensweisen nicht zu erwarten sind. Während der therapeutischen Behandlung müssen sich die Klient*innen in den moralischen Erwartungsrahmen der Organisation einpassen, damit zum Beispiel eine frühzeitige Entlassung auf Bewährung unterstützt wird (Weinberg 2000). Zudem kann die glaubhafte Bezugnahme auf das Konzept von Sucht-als-Krankheit auch Freiräume und Rechtfertigungsmöglichkeiten eröffnen, indem etwa schlechtes Verhalten entschuldigt oder irrationales erklärt werden kann. Es kann aber auch ein performativer Prozess einsetzen, in dem die Betreffenden ihre neu konstruierten Lebensgeschichten nach den grammatikalischen und syntaktischen Regeln des Krankheitsdiskurses, die sie gelernt haben, (nach-)erzählen (Reinarman 2005).

Personen, die wegen drogenbezogenen Delikten strafrechtlich verurteilt wurden, sind also aufgrund des ihnen zugewiesenen sozialen Status gefordert, sich mit dem gesellschaftlichen Anpassungsdruck hinsichtlich Resozialisierung und Rehabilitation zu arrangieren. Die im weiteren Verlauf hinzukommende Anforderung ist die Reintegration in die gesellschaftlichen Ordnungsvorstellungen jenseits der Straf- und Behandlungssysteme, insbesondere in den Arbeitsmarkt. An dieser Schnittstelle werden vor allem Substitutionsprogramme als wirksamer Stabilisator gesehen (Uchtenhagen 2010). Betreffende Personen erleben die Kontakte in den Abgabestellen aber nicht selten als stigmatisierend und meiden diese deshalb (Schmid et al. 2009). Durch die Teilnahme an solchen Programmen verschwinden vorherige moralische Zurechnungen und potenziell damit verbundene Kontroll- und Sanktionsarrangements nicht einfach, sondern verändern sich und verbinden sich mit anderen. So wird zum Beispiel der ehemals verfolgte und inhaftierte Junkie in den Status eines staatlich registrierten und kontrollierten Suchtkranken überführt (Schmidt-Semisch und Wehrheim 2007). Solche Statuswechsel bedürfen subjektiver Anpassungs- sowie Aneignungsleistungen und fordern den Betreffenden zusätzliche soziale Positionierungen ab. Die Subjekte müssen dann die jeweilige Konstellation ihrer »Mehrfachzugehörigkeiten « (Hirschauer 2014) beständig koordinieren.

Gerade Personen mit dem biografischen Hintergrund einer kriminellen Karriere sowie langjährigem intensiven Drogenkonsum sind entweder schon diskreditiert, oder 
zumindest aber diskreditierbar. Das heißt, sie sind immer wieder mit Fragen des Umgangs mit der eigenen Vergangenheit gegenüber anderen konfrontiert (Goffman 1975). Besonders bei der Arbeits- und Wohnungssuche kommt es zu Stigmatisierungserfahrungen, wenn etwa ein Strafregisterauszug verlangt und man deshalb abgewiesen wird.

Auch im Bereich der Arbeitsintegration, in der Schweiz etwa der Sozialhilfe und der Invalidenversicherung (IV), sind moralische Kategorisierungen in Kraft (Nadai 2009). Sie bestimmen mit über die Unterstützungswürdigkeit, und damit über den Zugang zum Hilfesystem. Hierbei müssen sich Hilfesuchende bei der Abklärung ihrer Bedürftigkeit quasi selbst diskreditieren, indem sie ihre Unfähigkeit zur selbstständigen Existenzsicherung nachweisen. Sozialhilfe und IV sind auf eine möglichst umfassende Integration in den ersten Arbeitsmarkt ausgerichtet und orientieren ihr Handeln an Aktivierungsstrategien, wodurch die Eigenverantwortung mittels Hilfe- und Kontrollmaßnahmen gestärkt werden soll. Leitend ist hierbei das Leistungsprinzip, das eine normative Hierarchisierung hinsichtlich der ökonomisch verwertbaren Arbeitsfähigkeit verfolgt und damit soziale Ungleich heiten legitimiert. Der moralische Vorwurf von Faulheit auf Kosten der steuerzahlenden Gemeinschaft ist dabei zentraler Bezugspunkt (Nadai 2006). Ganz unten in der Rangordnung stehen diejenigen mit den schlechtesten Aussichten, in reguläre und existenzsichernde Erwerbsarbeit (wieder) einzutreten. Durch die Ausblendung relativierender Aspekte wie etwa mangelnde Chancengleichheit kann »der starke Rekurs auf Eigenverantwortung [als] Freibrief für die Abwertung der Nichterfolgreichen « (Klein und Groß2011, 152) angesehen werden. Dieser Zuschreibung entkommt man auch dann nicht vollkommen, wenn man sich arbeitsfähig und leistungsbereit präsentiert, aber auf dem freien Markt nicht reüssieren kann. Stattdessen müssen zumeist Aktivierungsmaßnahmen absolviert werden, die strukturelle Widersprüche aufweisen, insbesondere durch die individualisierte Verantwortungszurechnung sowie existenzbedrohende Sanktionsmöglichkeiten (Nadai 2006 und 2009; Zahradnik 2018). Hieraus können psychosoziale Belastungen entstehen, die Integrationsprozesse möglicherweise durchkreuzen und zu einer verschärften sozialen Marginalisierung führen (Room 2005).

Durch die Erfahrung abwertender Zuschreibungen in den verschiedenen Bereichen können Selbstbilder erschüttert werden und kann das Bedürfnis wachsen, die eigene moralische Position zu stärken. Im Folgenden werden hierzu einige zentrale Forschungsergebnisse skizziert.

\section{Moralische Positionierungen und ihre subjektive Bedeutung}

Moralische Differenzkategorien haben mindestens ein Pendant (zum Beispiel kriminell vs. nicht-kriminell), sind in sich meist aber noch feiner gegliedert und werden mit sich 
historisch wandelnden Bedeutungen versehen. Aus einer Vielfalt an zirkulierenden Geschichten zu diesen Kategorien müssen die Einzelnen nun solche für sich finden, die dem Selbstsein möglichst Sinn verleihen und geeignet sind, die moralische Position zu stärken (Loseke 2007).

In Bezug auf Drogenkonsum bildet die Unterscheidung von kontrolliertem und unkontrolliertem Konsum auf diskursiver Ebene eine zentrale moralische Differenzierungslinie. Wenn man der übergeordneten kategorialen Zuordnung zu den Drogenkonsument*innen nicht gänzlich entkommen kann, ist es möglich, zumindest die Position der/des kontrollierten Konsumentin/Konsumenten für sich zu beanspruchen. Um sich von den unkontrollierten Konsument*innen und damit auch vom Stereotyp Junkie abzugrenzen, kann auf verschiedene Dimensionen wie äußeres Erscheinungsbild oder Konsumverhalten Bezug genommen werden. So lässt sich auch Anhänger*innen konventioneller Ansichten eine Unterscheidung der Konsument*innen vermitteln. Dies gelingt vor allem mit Bezug auf die Selbstkontrolle über den eigenen Konsum, im Erfolgsfall kann die Zuschreibung von Abhängigkeit geschwächt und somit auch Schamund Schuldgefühlen begegnet werden (Copes 2016). Die Intensität der Abgrenzungsbemühungen wird auch dadurch mitbestimmt, wie nahe man der stigmatisierten Gruppe schon rein äußerlich zurechenbar ist. Sozial integrierte Gelegenheitskonsument*innen müssen sich weniger Gedanken darüber machen, dass sie wegen ihrer Lebensführung missverständlich kategorisiert werden.

Bei Personen mit Therapieerfahrung sind dann wiederum andere Differenzkonstruktionen beobachtbar als bei denjenigen, die sich gerade in einer intensiven Konsumphase befinden. Erstere können bei der Distanzierung von unkontrollierten Konsument*innen die eigenen Erfahrungen quasi als besonders glaubhaften Beweis für die Zugehörigkeit zu den geheilten Suchtkranken anführen und dies narrativ in $\gg$ rock bottom stories « (Copes 2016, 210) oder » ruin-and-redemption «-Erzählungen (Reinarman 2005, 314) integrieren. In einer Abwärtsspirale geht es dabei bis hin zum Kollaps, der dann wiederum der Katalysator des eigenen Wandels ist, wobei es unterschiedliche Varianten dieser Geschichten gibt (Hänninen und Koski-Jännes 1999; Pienaar und Dilkes-Frayne 2017).

Auch in Bezug auf Kriminalität lassen sich symbolische Grenzziehungen beobachten, die bestimmte Werte und Normen vermitteln. Durch die Unterscheidung eines moralisch guten Ich/Wir von einem abweichenden Die wird bekräftigt, wer man ist und wer man nicht sein will (Sandberg 2016). Hierbei gilt vor allem die moralische Beurteilung der Schwere von Vergehen als Richtschnur für Differenzierungen (Sandberg et al. 2015). In der Desistance-Forschung ${ }^{1}$ werden die Narrative von strafrechtlich Verurteilten untersucht, die für sich häufig einen inneren Wandlungsprozess reklamieren und sich dadurch von ihrem früheren kriminellen Ich distanzieren (Maruna 2001). Ein zentrales Konzept ist das »knifing-off « (Maruna und Roy 2007), 
mit dem das umfassende Abschneiden des alten Lebens und die daran anschließende Identitätstransformation gemeint ist. Im Rahmen von Desistance-Narrativen finden sich aber immer wieder auch Neutralisierungstechniken, wie etwa die Verschiebung von Verantwortung und Schuld auf andere Personen oder soziale Umstände (Maruna und Copes 2005). Gegenüber den Vertreter*innen von Strafverfolgungsbehörden und Vollstreckungseinrichtungen macht es so in vielerlei Hinsicht einen Unterschied, ob man sich etwa verantwortungsbewusst, reumütig und veränderungsbereit präsentiert oder nicht. Insgesamt wird die Anerkennung des eigenen Wandels durch andere als ein zentraler Erfolgsfaktor für den Desistance-Prozess angesehen (Maruna et al. 2004).

Bei der Arbeitsintegration ist vor allem die Leistungsnorm maßgeblicher Bezugspunkt für moralische Differenzsetzungen. Im Kontext von Arbeitslosigkeit droht die stigmatisierende Fremdzuschreibung von Faulheit das Selbstbild anzugreifen, woraufhin die Umlenkung dieser moralischen Kategorie auf andere Arbeitslose sowie die Selbstpositionierung als arbeitswillig eine entlastende Reaktionsweise darstellen kann (Zahradnik 2018). Eine andere Variante kann die Übernahme von Vorurteilen gegenüber weiteren Gruppen sein, die als bedrohliche Konkurrenz bei der Verteilung knapper Ressourcen angesehen werden.

\section{Methodisches Vorgehen}

Die hier untersuchten Längsschnittinterviews sind in einem größeren Projektzusammenhang ${ }^{2}$ entstanden, in welchem die Reintegrationsprozesse von strafrechtlich verurteilten Männern in der Deutschschweiz untersucht werden. Die Interviewpartner befanden sich zum Zeitpunkt des ersten Interviews zumeist noch am Ende einer Inhaftierung oder einer von der Justiz angeordneten stationären Maßnahme. In der ersten Welle wurden mit 50 Männern im Alter von 16 bis 61 Jahren teilstrukturierte narrative Interviews erhoben, mit 30 Personen konnten insgesamt vier Interviews im Abstand von etwa eineinhalb Jahren wiederholt geführt werden (Zahradnik et al. 2019). In den Interviews der ersten Welle standen neben der Lebensgeschichte vor allem die Erfahrungen der institutionellen Unterbringung sowie die Pläne für die Zeit nach deren Beendigung im Vordergrund. Die Folgeinterviews waren dann zuerst retrospektiv auf die Zeit seit dem letzten Gespräch gerichtet, wobei nach dieser offenen Erzählaufforderung verschiedene thematische Bereiche wie zum Beispiel Wohnsituation, Partnerschaft, Familie usw. angesteuert wurden, und schlossen mit einem Zukunftsausblick ab.

In der fallübergreifenden Auswertung, die sich an den Codierschritten der Grounded Theory Methodology (Strauss und Corbin 1996) orientierte, zeigten sich in 
einigen Fällen deutliche moralisch gefärbte Positionierungsaktivitäten. In diesen Interviews wurden zumeist auch Stigmatisierungserfahrungen aufgrund von (ehemaligem) Drogenkonsum und Vorstrafen geschildert. Die Interviews des hier untersuchten Falles erschienen in dieser Hinsicht aufgrund der thematischen Dichte sowie der im Längsschnitt beobachtbaren Dynamik besonders aufschlussreich für eine explorative Einzelfallanalyse.

Die Erzählungen über vier Interviews hinweg wurden in Anlehnung an die Methode der »theorieorientierten Fallrekonstruktion « (Miethe 2014) analysiert, wobei das Datenmaterial vor allem hinsichtlich des theoretischen Vorwissens und der Relevanz für die Fragestellung gewichtet wurde. Theorie hatte hier aber eine heuristische Funktion und sollte die Auswertung anleiten und inspirieren, keinesfalls dominieren. Es galt also, theoretische Vorannahmen nicht einfach bruchlos an das Material anzulegen, sondern im Wechselspiel mit der Empirie weiterzuentwickeln. Hierbei musste auch das Verhältnis zwischen den an der Forschungssituation Beteiligten sowie der Untersuchungskontext mit seinen normativen Deutungsangeboten beständig im Blick behalten und kritisch reflektiert werden (Bereswill und Rieker 2008).

Die im Folgenden analysierten Textstellen wurden vor dem Hintergrund des oben aufgespannten theoretischen Rahmens ausgewählt. In die engere Auswahl gelangten so Interviewpassagen, die moralische Selbst- und Fremdpositionierungen sowie einen inhaltlichen Bezug zu Drogenkonsum, Arbeit und Kriminalität aufwiesen. Sie wurden im nächsten Schritt einer genaueren »Positionierungsanalyse « (Lucius-Hoene und Deppermann 2004) unterzogen, um Prozesse der Dar- und Herstellung narrativer Identität zu rekonstruieren. Ein Großteil des Erkenntnispotenzials von Selbsterzählungen liegt in ihrer »Ambiguitätsstruktur « (Hanses 2010, 95), also der grundsätzlichen und widerspruchsvollen Verwobenheit von Subjekt und Lebenswelt, Eigensinn und sozialer Strukturiertheit, Veränderung und Beständigkeit sowie Vergangenheit und Gegenwart. In den Interviews wurde deshalb untersucht, in welcher Art und Weise sich der Erzähler seiner eigenen Lebensgeschichte sowie gesellschaftlichen Diskursen zu Drogen, Kriminalität und Arbeit zuwendet und wie sich dabei sein Selbst- und Weltverhältnis konstituiert.

Ein zusätzlicher Erkenntnisgewinn der hier vorgenommenen Einzelfallanalyse liegt vor allem darin, dass sich durch die Längsschnittbetrachtung die moralischen Positionierungsaktivitäten im Zeitverlauf untersuchen lassen. Somit können »Wandlungen, Ambiguitäten und (In)konsistenzen von Orientierungen und Handlungen in der Auseinandersetzung mit sich verändernden gesellschaftlichen Rahmenbedingungen oder Situationen « (Witzel 2020,14) analysiert werden. Der Ansatz erlaubt zudem, »Biografisierungen in Form von nachträglichen Sinnzuschreibungen und Umdeutungen nachzuweisen und ihre Bedeutung zu analysieren « (ebd.). 


\section{Ergebnisse der Einzelfallanalyse}

Bevor die vier Interviewerzählungen eingehend analysiert werden, wird ein kurzer Überblick der Fallgeschichte gegeben, wie sie vom Gesprächspartner dargelegt wurde. Die Darstellung innerhalb der Analyse der einzelnen Interviews ist so aufgebaut, dass die jeweils zentralen moralischen Positionierungsmuster in den Bereichen Arbeit, Drogenkonsum und Kriminalität behandelt werden. Die Muster sind thematisch aber nicht trennscharf, sondern weisen immer wieder Überschneidungen auf.

\subsection{Fallgeschichte}

Vor dem ersten Interview wurden die Studienteilnehmer gebeten, sich einen frei gewählten Codenamen zu geben, mit dem sie dann sogleich im Gespräch angesprochen wurden. Hierdurch sollte das Datenmaterial schon während der Erhebungssituation in einem ersten Schritt anonymisiert werden. In einigen Fällen zeigte sich aber auch, dass diese Codenamen erste Spuren auf die subjektive Bedeutungszuschreibung der eigenen Biografie enthielten. Der Mann, dessen Erzählungen hier analysiert werden, hat sich den Namen »Zickzack « gegeben, wodurch das Bild eines unsteten Lebensverlaufs mit abrupten Richtungswechseln transportiert wird.

Zickzack erzählt, dass er Anfang der 1970er Jahre in der Schweiz geboren und zum Zeitpunkt des ersten Interviews im Jahre 2013 Anfang 40 ist. Im Alter von 16 Jahren habe er zum ersten Mal Drogen genommen und sei in den folgenden Jahren Teil der offenen Heroinszene ${ }^{3}$ gewesen. Er habe zwei Ausbildungen absolviert, wobei er den niedrigen Lehrlingslohn durch den Handel mit Drogen kompensiert habe. Anfang der 1990er Jahre sei er zum ersten Mal verhaftet und inhaftiert worden. In den darauffolgenden Jahren habe er verschiedene Entzugsversuche unternommen. Anfang der 2000er Jahre habe er sich für 18 Monate in eine Drogentherapie begeben, sei anschließend aber wieder ins alte Umfeld zurückgekehrt und habe intensiv konsumiert und mit Drogen gehandelt. Nach einigen Jahren habe sich der Wunsch nach einem Ausstieg aus Konsum und Handel wieder gefestigt, er habe sich aus den Strukturen aber nicht lösen können. Schließlich sei er von der Polizei überwacht und verhaftet worden, woraufhin er für mehrere Monate in U-Haft gemusst und seine Wohnung verloren habe. Er sei zu mehreren Jahren Gefängnis verurteilt worden, aufgrund seiner Kooperationsbereitschaft und seines als krankhaft eingestuften Drogenkonsums sei statt der Haftstrafe aber ersatzweise eine mehrjährige Drogentherapie angeordnet worden. Er habe auch eine Drogenersatztherapie begonnen und seitdem kein Heroin mehr konsumiert. Zum Zeitpunkt des ersten Interviews wohne er in einer an die Therapieeinrichtung angegliederten Wohnung, wodurch der endgültige Austritt vorbereitet werden solle, und versuche eine Stelle auf dem ersten Arbeitsmarkt zu finden. 


\subsection{Erstes Interview}

\section{Arbeitsorientierung trotz Drogenkonsum}

Im ersten Positionierungsmuster werden Arbeitsorientierung und Drogenkonsum eng aufeinander bezogen. Die Bekräftigung, trotz Heroinkonsums »immer gearbeitet $\ll$ zu haben, zieht sich durch das gesamte Interview. In einer Passage wird dies in einer reinszenierten Erwiderung auf die Kritik der Eltern am früheren Lebensstil dargestellt:

»Wenn meine Eltern oder meine Mutter wieder etwas gesagt haben, da sagte ich: $>$ Schau, ich arbeite, ich bezable Steuern, ich zable dies und jenes, lasst mich doch machen. Ich schade mir, ich konsumiere halt, aber ich bin ein Teil der Gesellschaft. $\ll{ }^{4}$

Das frühere Ich wird hier als sozial integrierter, weil kontrollierter Konsument präsentiert, der seinen bürgerlichen Pflichten in Form von Erwerbsarbeit nachkommt, wodurch die konsumbedingte Selbstschädigung moralisch gerechtfertigt wird. Unterstrichen wird dies durch die lebhafte Darstellung in direkter Rede.

Diese Positionierung wird an mehreren Stellen im Interview durch wertende Abgrenzungen von anderen Drogenkonsument ${ }^{*}$ innen bekräftigt:

»Für mich war ein Süchtiger ein [Orstname]-Typ. Das sind für mich diese Leute gewesen, welche ich nicht sein wollte, also mich nicht so tief sinken lassen wollte und bis heute hat mich das am Leben erhalten und ich habe auch immer geschaut auf Sauberkeit, eine Wohnung, dass man Duschen kann und ein Bett hat.«

Bezugspunkt ist hier eine regionale Variante des Stereotyps eines Junkies, der für die unterste Stufe in der Hierarchie der Drogenkonsument*innen steht, denn sie hätten die Kontrolle über ihren Konsum und ihre Lebensführung verloren. Ihnen wird die Funktion eines abschreckenden Beispiels zugeschrieben, das über die Zeit bis in die Gegenwart ein Abgleiten verhindert habe. Hier wird die statussichernde Funktion von abgrenzenden Kategorisierungen explizit deutlich.

Auch in Bezug auf die Arbeit im Beschäftigungsprogramm der Therapieeinrichtung wird die eigene Arbeitsorientierung hervorgehoben: »Das hat auch Frau [Name] von der Maßnahme gesehen [...]. Ich bin einer, der zuverlässig sein Zeug macht und wütend werde, wenn die Anderen das Gefühl haben, sie müssen es nicht machen. « Die Betreuerin wird hier als bekräftigende Referenz der eigenen Positionierung als pflichtbewusster Arbeiter angeführt. Demnach habe auch sie die Defizite der anderen Teilnehmer*innen hinsichtlich Arbeitsfähigkeit ( $\gg$ die haben noch nie gearbeitet «), aber auch grundle- 
gender Regeln der Körperhygiene (»nicht fähig, jeden Abend zu duschen «) erkannt. Für sich selbst wird eine höhere Stellung in der moralischen Hierarchie des therapeutischen Stufenkonzepts in Anspruch genommen, weshalb es gerechtfertigt sei, dass er die »Leute ein wenig leitet «. Dies habe aber keine Erfolge gebracht und führe seinerseits zu Unzufriedenheit, woraufhin eine Betreuerin der Einrichtung bekräftigend gesagt habe: » [Zickzack], du kannst dich nicht als Maßstab für alle anschauen.<« Zusammengenommen wird hier eine moralische Grenze gezogen, die zwischen denjenigen trennt, die fähig sind, Arbeit und Drogenkonsum so weit in Einklang zu halten, dass eine bestimmte Vorstellung von Lebensführung realisiert werden kann, und solchen, denen dies nicht gelingt.

\section{Drogentherapie als Erfolg}

In Bezug auf den Drogenkonsum wird eine Positionierung als erfolgreicher Therapieteilnehmer sichtbar: »Ich habe es jetzt auch nach 20 Jahren im zweiten Anlauf geschafft.«Um den Erfolg zu sichern, soll zu den früheren Orten und Personen, denen ein schlechter Einfluss zugesprochen wird, Abstand gehalten werden. Eine Stärkung erfährt das Positionierungsmuster, wenn es darum geht, die Schuldfrage für den eigenen Konsum zu klären. Anhand einer Gruppentherapiesitzung wird exemplarisch aufgezeigt, dass die anderen Teilnehmer*innen immer wieder nach externen Erklärungen für ihr Konsumverhalten suchen würden: »Wenn das Gespräch auf Drogensucht kommt, sagt jeder: >Ja, der ist schuld.<« Dieses Vorgehen wird aber als unzulässig zurückgewiesen, denn es sei »Charaktersache«, dass man zu seinen früheren Konsumentscheidungen stehe, denn »jeder kann nein sagen «. Diese Betonung von Eigenverantwortung wird aber brüchig, wenn direkt im Anschluss narrative Anknüpfungspunkte in der Phase von eigenen erfolglosen Entzugsversuchen gesucht werden.

Eine wichtige Stütze für die aktuelle Situation und den weiteren Fortgang wird in der mittlerweile mehrjährigen Substitutionsbehandlung gesehen: »Aber so wie ich jetzt lebe, mit dem Subutex, mit dieser Sicherheit, will ich in zwei Jahren gleich sein wie jetzt. « Dem Substitut wird zuerkannt, unerwünschte affektive Reaktionen auf stressvolle Reize zu unterbinden und die Gefahr der Wiederaufnahme alter Konsumgewohnheiten zu verhindern. Hierdurch wird die Position als anerkannter und angepasster Suchtkranker moralisch bekräftigt.

\section{Distanzierung von Kriminalität}

Die Jahre des intensiven Drogenkonsums und -handels sind in den Erzählungen mit unterschiedlichen Facetten ausgestattet. Auf der einen Seite werden die lustvollen und 
abenteuerlichen Momente eines durch vielfältige Kontakte und Reisen geprägten Lebensstils herausgestellt, der durch den Drogenhandel problemlos finanziert werden konnte. Auf der anderen Seite aber stehen die unzähligen erfolglosen Versuche, den Konsum zu beenden, sowie die strukturellen Restriktionen, aus dem Drogenhandel auszusteigen:

»Weil am Schluss brauchte ich 15 Gramm Heroin am Tag und fast 10 Gramm Koks, bevor sie mich verhaftet haben. Darum bin ich auch auf die Idee gekommen, dass ich aussteigen will. Ich habe gemerkt, es wird mir zu viel und ich habe gewusst, ich will nicht mehr. Die Polizei ist halt früher gewesen als ich freiwillig, aber vielleicht hat es das auch gebraucht.«

Ausgehend von der täglichen Menge an Drogen, die konsumiert wurden, wird der entscheidende Wendepunkt eingeführt. Demnach sei der Wunsch nach einem Ausstieg schon vorher gereift, wodurch die eigene moralische Position aufgewertet wird. Die Polizei sei der Umsetzung aber durch die Verhaftung zuvorgekommen. Hiernach wird der polizeiliche Zugriff als positiver und notwendiger Schicksalsschlag ausgedeutet, der neue Handlungsoptionen eröffnet habe.

Es schließt sich eine Geschichte der engen Kooperation mit der Polizei an, in der die Position eines wertvollen und bereitwilligen Informanten vertreten wird (»Wir haben in ein Wespennest gestochen «). Aus der Zusammenarbeit sei zudem eine anhaltende freundschaftliche Beziehung mit einem der Ermittler entstanden. Durch diese deutliche Nostrifizierung mit den Strafverfolgungsbehörden wird der eigene moralische Standpunkt weiter gestärkt.

\subsection{Zweites Interview}

\section{Fehlkategorisierungen im Kontext der Arbeitsintegration}

Eine bedeutende Veränderung der Situation habe sich mittlerweile durch den Wechsel der primären Fallzuständigkeit hinsichtlich der Arbeitsintegration ergeben. Diese habe vorher ausschließlich bei der Justiz und der Therapieeinrichtung gelegen und sich nun hin zum Sozialamt und der IV verlagert. Aus der neuen Zuständigkeit habe die zwangsweise Herausnahme aus dem Beschäftigungsprogramm der Therapieeinrichtung resultiert. Insgesamt drehen sich viele Passagen des Interviews um »Fehlkategorisierungen « (Hirschauer 2017, 40) im Behördenkontext. Die Arbeitsweise des Sozialamts wird so dargestellt, dass in Bezug auf die Teilnahme an einer Arbeitsintegrationsmaßnahme Druck durch Sanktionsdrohungen aufgebaut werde, zugleich aber 
das Passungsverhältnis zwischen Teilnehmer und Maßnahme keine ausreichende Beachtung finde:

»Also ich habe der vom RAV [Regionale Arbeitsvermittlung] letzte Woche gesagt: >Sie Frau [Name], da wo sie mich hingeschickt haben, habe ich gemeint, es sei eine Schnittstelle erster Arbeitsmarkt, aber das ist für mich irgendwie sechster Arbeitsmarkt, was hier läuft.<«

Die der Behörde angelastete Fehlkategorisierung und die damit verbundene Betroffenheit werden durch eine begriffliche Übertreibung betont, indem die Diskrepanz zwischen der Selbstverortung (»Schnittstelle erster Arbeitsmarkt «) und der Fremdverortung (»sechster Arbeitsmarkt «) sehr groß gestaltet wird. Hierdurch drückt sich der Unmut über die Fremdbestimmung aus, die einen als positiv erachteten Verlauf jäh unterbrochen habe: »Ich habe gerade eine gute Welle erwischt, es ging mir gut äh ja, es ist einfach, es hat sich gut angefühlt äh wie das Leben gelaufen ist und dann hat es geheißen: > Ja nein, jetzt müssen sie trotzdem. $<\ll$

Eine ähnliche Situation wie im RAV wird auch im Kontext der IV-Abklärung beschrieben, als es um die Berechtigung geht, ob zusätzliche Unterstützungsleistungen wegen körperlichen Beschwerden gewährt werden:

$\gg$ Da hatte es einen Arzt und [...] der hat mir gerade an den Kopf gesagt: >Ja wegen Sucht gäbe es keine IV.< [...] Er hat mir die ganze Zeit ein wenig zu verstehen gegeben: > Ja einen Giftler, den brauchen wir hier nicht.< Bin dann irgendwann mal sauer geworden, [...] ich hätte jetzt auch das Leben lang gearbeitet trotz dem Gift.«

Folgt man der vorgebrachten Darstellung, dann argumentiert der Amtsarzt in einem Bezugssystem, in dem Sucht nicht als Krankheit, sondern als selbstverschuldete Eigenschädigung angesehen wird und damit den Ausschluss aus dem Kreis der Leistungsberechtigten begründet. Dies steht in deutlichem Kontrast zum Positionierungsmuster des arbeitsfähigen Drogenkonsumenten, weil der Arzt nicht der moralischen Logik folgt, dass harte Arbeit selbstschädigenden Drogenkonsum legitimieren würde.

\section{Therapiemüdigkeit und erneuter Konsum}

Die bisherige Dauer der Unterbringung in der Einrichtung wird mit fünf Jahren angegeben, üblicherweise liege sie aber nur bei zwei Jahren. Dies führe mit dazu, dass sich eine zunehmende Unzufriedenheit einstelle:

»Ja, es nagt an einem diese Unselbständigkeit, weil ich bin nicht mehr 20 und eben, es gibt Leute, die sind 15 Jahre jünger als ich und haben das Gefühl sie müssen mir sagen wie das 
Leben aussieht und solche Leute kann ich nicht ernst nehmen. [...] Der muss mir zuerst einmal beweisen, dass er mehr drauf hat als ich. Ich meine ich habe schon wirklich vieles erlebt und ich habe alles überstanden irgendwie und manch einer von diesen Globis dort würde wahrscheinlich in meiner Situation den Bettel hinwerfen.«

In dieser Sequenz wird eine Differenzkonstruktion nach Alter beziehungsweise Lebenserfahrung eingeführt, die mit der Machtdifferenz zwischen Teilnehmer und Betreuungsperson im Therapiekontext verbunden wird. Die Begrenzungen der Lebensführung werden demnach illegitimerweise von Leuten durchgesetzt, die jünger und damit unerfahrener sind. Die eigene Lebenserfahrung wird noch einmal als biografische Ressource aufgewertet, indem eine direkte Vergleichssituation konstruiert wird. Durch den in der Schweiz als mildes Schimpfwort gebräuchlichen Begriff »Globi« werden die Betreuungspersonen kollektiv abgewertet und die eigene moralische Position gestärkt.

Weiter seien zunehmend Gefühle aufgekommen, »therapiemüde « und »fremdbestimmt « zu sein. Zusammen mit einem verschlechterten Gesundheitszustand und Schmerzen sei es zu einer Wiederaufnahme des Kokainkonsums gekommen. Dieser sei zwischenzeitlich etwas außer Kontrolle geraten (»ein wenig zu weit aus dem Fenster raus gewagt $\ll$ ) und es sei $»$ nicht so einfach geworden $\ll$, ihn wieder in den Griff zu bekommen. Aber ein gänzlicher Kontrollverlust wird mit Verweis auf die Aufrechterhaltung der zentralen Wertmaßstäbe von Arbeit und Pflichterfüllung zurückgewiesen (»ich bin trotzdem immer arbeiten gegangen, habe meine Sachen erledigt «), wodurch die moralische Position wieder gestärkt wird.

Diese Erfahrung habe zudem Unsicherheiten über die Ursachen des eigenen Konsumverhaltens mit sich gebracht ( $\gg$ ich kann das nicht erklären, wieso und warum es passiert. Zäck und schon habe ich es gekauft «). Eine Erklärung soll die medizinische Methode des »Neuro-Feedback« liefern, bei der neurologische Hirnströme analysiert werden. Ihr wird die Funktion zugeschrieben, die Ursachen erklären und steuernd auf das Verhalten einwirken zu können. Hier zeigt sich wieder die Positionierung als angepasster Suchtkranker, der die Verantwortlichkeit für das eigene Handeln aber in weiten Teilen an eine medizinische Experteninstanz auslagert.

Diese Selbstpositionierung wird ergänzt zu der schon im ersten Interview präsentierten Positionierung hinsichtlich der Verantwortungsübernahme für das eigene Konsumverhalten. Den Referenzpunkt des Vergleichs bildet die zurückliegende Zeit, in der eine Position als selbstbestimmter Konsument entworfen wird (» weil ich es wollte $\ll)$. Es wird vor diesem Hintergrund aber weiterhin vertreten, dass es keine haltbaren Belege dafür gebe, dass eine belastete Kindheit oder der sozioökonomische Hintergrund einen entscheidenden Einfluss auf den späteren Konsum habe: »Ich finde es eh schade, wenn Leute sagen: >Ja, wegen der Kindheit und Zeug.< Weil konsumieren das tust du immer noch selber.« Ein Widerspruch ist nun darin zu erkennen, dass einerseits 
der Standpunkt vertreten wird, dass der Konsum Ergebnis einer bewussten Entscheidung ist, andererseits aber die eigenen Konsumentscheidungen nicht erklärbar sind.

\section{Belastungen und Delinquenzgedanken}

Die aktuelle Lage wird aufgrund nur geringer Sozialleistungen als zunehmend belastend beschrieben und mit dem Entwurf in Verbindung gebracht, dass strafbare Handlungen deshalb wieder eine Option sein könnten (»Es ist schwierig, dass ich nicht mehr delinquent werde«). Die positiv konnotierte Vergangenheit, als durch den Drogenhandel noch ausreichend Mittel zur Verfügung standen, fungiert nun als Referenzpunkt, vor dem die derzeitige Lebenslage abfällt. Hier wird deutlich, wie die moralische Positionierung als gesetzestreu durch die vielfältigen Einschränkungen in der aktuellen Situation herausgefordert wird und ins Wanken gerät.

\subsection{Drittes Interview}

\section{Wiedererstarken im Arbeitskontext}

In der Zwischenzeit sei die Arbeitsmaßnahme des Sozialamtes beendet und die Arbeit im Beschäftigungsprogramm der Therapieeinrichtung wieder aufgenommen worden. Weiterhin wird der erste Arbeitsmarkt als Ziel ausgegeben: »Jetzt bin ich dort dran, wieder einen Job zu suchen. « Die Arbeit im Beschäftigungsprogramm wird trotz des niedrigen Lohns, der durch Sozialleistungen aufgestockt werden muss, als sinnstiftend entworfen. Auch hier zeigt sich wieder die Positionierung als arbeitswillig, was durch die Abgrenzung von anderen Leistungsempfänger*innen bekräftigt wird:

»Ich meine, die meisten äh, wo so Geld bekommen, die machen gar nichts oder finden: $>$ Jaja, eben ich gehe es verspielen oder irgendwie krank.< Mir ist das zu blöd gewesen, weil ich mache es für mich, ich schaffe gerne, das erhaltet mich, ja.«

Unter ihnen wird eine Mehrheit ausgemacht, der die Position des leistungslosen Transferbezugs zugeschrieben wird. Für sich selbst hingegen wird beansprucht, nicht des Geldes wegen, sondern aufgrund einer gefestigten Arbeitsmoral tätig zu sein.

An anderer Stelle dienen die früheren Weggefährt*innen als negative Vergleichsfolie bezüglich der Leistungsbereitschaft:

»Wenn ich die anschaue und dann den Vergleich zu mir ziehen muss, weil ich muss ja auch ein bisschen für mich Pluspunkte sammeln [...]. Die einen, ich meine, das sind, das 
sind Kleindealer, die vermitteln schon seit, seit ich die kenne, die haben schon für mich geschafft, und die haben nie einen Aufstieg gehabt. [...] Es schreckt mich ab, wenn ich die Leute heute noch sehe, und es bestärkt mich in dem, dass es funktioniert.«

Hier wird explizit die Funktion des angestellten Vergleichs präsentiert, nämlich die Aufwertung der eigenen Position. Es wird eine Differenz zu alten Weggefährt*innen aufgestellt, die damals schon für ihn gearbeitet, sich aber nie leistungsorientiert gezeigt hätten. Im Gegensatz zu ihm hätten sie deshalb keinen Aufstieg in der Statushierarchie des Drogenhandels erreichen können. Bemerkenswert ist an dieser Sequenz, dass der Drogenhandel hier zum ersten Mal mit Arbeit in Verbindung gebracht wird ( $\gg$ für mich geschafft $\ll)$. Am Ende wird das abschreckende Potenzial der Differenzkonstruktion sowie deren identifikationsstiftende und förderliche Funktion hinsichtlich der Erreichung der ausgegebenen Ziele noch einmal deutlich hervorgehoben.

\section{Beendeter Konsum und äußerer Zuspruch}

Die Positionierung als erfolgreicher Therapieteilnehmer wird in der Gesamterzählung an mehreren Stellen durch äußeren Zuspruch von verschiedenen Betreuungspersonen gestützt: »Ich mag gar nicht mehr. Mein Chef hat es heute auf den Punkt gebracht, hat gesagt: > Gell du bist nicht mehr [...] auf der Überholspur.<« Hier wird ein Deutungsrahmen aufgespannt, in dem der sich selbst zugeschriebene Ermüdungsprozess bezüglich des früheren Lebens metaphorisch nun im Bild einer Autobahn ausgedeutet werden kann. Der damalige Pfad (»Überholspur «) konnte demnach verlassen und die Geschwindigkeit des Lebensstils angepasst werden.

Die Position des kontrollierten Konsumenten wird nun mit Bezug auf verschiedene Substanzen wieder gestärkt. So habe der zwischenzeitliche Kokainkonsum, der im zweiten Interview thematisiert wurde, beendet werden können (»Für mich ist das Thema wieder abgehakt «), was anhand von Situationen, in denen Angebote ausgeschlagen worden seien, unterstrichen wird (»Habe ich gesagt: $>$ Du nein, ist schon gut. $<\ll)$. Die erlangte Willenskraft wird als Errungenschaft eines langen Entwicklungsprozesses gekennzeichnet (»Die Stärke, die habe ich auch nicht von heute auf morgen gehabt.«) und durch die Distanzierung von den früheren Weggefährt*innen weiter aufgewertet (»Es gibt Leute, wo mich erkennen, und das ist dann eklig, wenn sie dir dann nachlaufen $\ll)$.

Zudem habe der Heroinkonsum mittlerweile seit mehreren Jahren beendet werden können, was eine »Genugtuung « mit sich gebracht habe (»Also für mich bin ich geheilt «). Lediglich Cannabis werde momentan noch konsumiert, wobei dies als unbedenklich angesehen wird. Hierfür werden verschiedene Betreuungspersonen aus Therapie und Justiz angeführt, die erkannt hätten, dass dies keine Rückfallgefahr in 
den Heroinkonsum darstelle, weshalb der gemäßigte Konsum (»nicht jeden Abend «) gestattet werde ( $\gg$ offiziell vom Bewährungsdienst und allen «).

Es zeigt sich aber an mehreren Stellen, dass die Position des kontrollierten Konsumenten immer wieder auch herausgefordert wird. So sei es nicht immer einfach, sich von früheren Bekannten, die noch konsumieren, fernzuhalten: »Ich darf wirklich nicht mehr in Versuchung kommen, also eben ich, ich werde das Leben lang süchtig sein und immer die Dämonen in mir drin haben, ja.«

Hier wird deutlich auf den Diskurs zu Sucht-als-Krankheit Bezug genommen, indem Sucht als ein nicht endgültig zu überwindender Zustand dargestellt und im Sinne einer dämonischen Macht ausgedeutet wird. Das ständige Ringen mit diesen Kräften sei darüber hinaus mit großen Anstrengungen verbunden, die auch immer wieder bis an den Rand der Erschöpfung führen würden.

\section{Reorientierung an Normalitätsvorstellungen}

Die Zugehörigkeitskonstruktion zu den Nichtkriminellen wird auch in diesem Interview wieder durch die Erzählung über die frühere Kooperation mit der Polizei unterstrichen. Zudem wird ein allgemeiner Wandlungsprozess beschrieben: »Ich habe es glaube begriffen, dass ich auch legal oder äh bei der Gesellschaft kann alt werden.« Die Orientierung an gesellschaftlicher Normalität wird wieder als gefestigt präsentiert. Dies wird dadurch bekräftigt, dass auch Angeboten eines Wiedereinstiegs in den Handel problemlos widerstanden werden könne (»>Willst du nicht Hasch oder Gras verkaufen? $<$ Dann sage ich: $>$ Nein. $<\ll)$.

Lediglich an solchen Tagen, an denen die Belastungen der Lebenssituation aufgrund bestimmter Einschränkungen bewusst würden, kämen stellenweise Gedanken auf, die das Gefängnis als Schonraum (»muss nichts aushalten «) erscheinen lassen: »Gibt es Tage, da denke ich: > Hey weißt was, schleckt mich alle am Arsch, am liebsten würde ich wieder in die Kiste, fertig. $<\ll$

\subsection{Viertes Interview}

\section{Aufstieg im Arbeitskontext}

Mittlerweile habe seit einigen Monaten der Status eines fest angestellten Arbeiters erlangt werden können, was nun eine andere Sprecherposition mit sich bringt. Große Teile des Interviews drehen sich thematisch um staatlich verordnete Kürzungen bei der Finanzierung von Hilfen für Drogenkonsument*innen, was konkret auch in der Therapieeinrichtung beobachtet worden sei. Es zeigen sich nostrifizierende Po- 
sitionierungen, wenn es um die Notwendigkeit von Anerkennung durch Arbeit im Beschäftigungsprogramm geht: »Wir brauchen das ein wenig, dass wir gebraucht werden oder etwas können machen.« Dies wird mit Überlegungen verbunden, welche Gruppen einen legitimen Anspruch auf Unterstützung hätten und wer diese zuerst erhalten sollte:

»Das sind Sachen, die mich dann manchmal sauer machen. Schau, [...] ich bin kein Nazi, aber gottverdammt, man gibt Geld aus für Integration und alles, aber 100.000 oder 150.000 Franken für Leute, die gestrauchelt sind, da spart man dann.«

Die Selbstpositionierung als Advokat der Unterstützungsbedürftigen bringt nun neue Differenzierungsaktivitäten mit sich, die auf eine Hierarchisierung nach Kriterien der nationalen Zugehörigkeit abstellen. An anderer Stelle werden aber wiederum Bekundungen der Nähe zu Personen mit Migrationshintergrund eingestreut, etwa in Bezug auf die neue Arbeitsstelle: »Ich meine, ich schaffe jetzt in einer Firma, 60 Leute, 95 Prozent sind Bosnier, coole Leute, die haben mich aufgenommen wie wenn ich einer von ihnen wäre.«

\section{Kontrollierter Kokainkonsum}

Bezüglich des Drogenkonsums ist die Positionierung teilweise in Bewegung geraten. Weiterhin werden die eigenen Erfolge durch die Argumentationsfigur der »Charaktersache « sowie die Abgrenzung gegenüber den unkontrollierten Konsument*innen hervorgehoben. Der Konsum von Heroin sei auch weiterhin überwunden und die Lage stabil (»Seit acht Jahren, eben kein Braunes mehr genommen, also da bin ich über dem Berg «). Im Kontrast zum vorherigen Interview wird nun aber eine Selbstpositionierung als kontrollierter Konsument von Kokain deutlich:

»Dann auch mein Betreuer in der Außenwohngruppe [...] hat das akzeptiert, dass ich halt ab und zu mal einen Absturz habe mit Weißem, also mit Kokain. Mein Gott. Ich sage nein, ich sage nicht Absturz, ich sage nicht Rückfall, einfach mal ein Konsumvorfall.«

Die Legitimität des neuen Konsumverhaltens wird dadurch gestärkt, dass es auch von außen Akzeptanz erfährt. Bemerkenswert ist die Zurückweisung der gebräuchlichen Begrifflichkeiten »Absturz« und »Rückfall «. Stattdessen wird der Begriff »Konsumvorfall « eingeführt, der auch eine moralische Entlastung in Hinblick auf die Konsumhandlung transportiert. 


\section{Moralische Unterschiede von Straftaten}

Weiterhin wird die Positionierung als grundsätzlich gesetzestreu durch die Nostrifizierungsgeschichte über die Kooperation mit der Polizei sowie der sich daraus entwickelten Freundschaft gestützt. Es zeigt sich nun aber ansatzweise auch eine kritische Selbstreflexion hinsichtlich des früheren Drogenhandels, die aber zugleich durch einen moralischen Vergleich mit den Vergehen des Großkapitals abgeschwächt wird: »Ich habe es verdient, ich habe jeden Tag verdient. Eigentlich, weil ich hätte noch viel mehr verdient, was ich alles gemacht habe. Aber die kommen meistens straffrei davon. « Die »Banken « seien die »größten Banditen «, denn sie » haben Anleger abgezockt « und würden in einem System der »Zweiklassengerechtigkeit « bevorzugt. Das Handeln der Wirtschaftskriminellen sei »nicht einfach Betäubungsmittelverkauf «, sondern sie nehmen »den Leuten ihr Erspartes weg« und »machen alles kaputt $\ll$.

\section{Fazit}

Es konnte gezeigt werden, dass innere Konflikte herausgefordert werden und sich zuspitzen, wenn die subjektive Wahrnehmung des eigenen sozialen Status verunsichert wird, also Statuswunsch und -wirklichkeit auseinanderfallen (vgl. Neckel 1991). Als Reaktion auf diese Konflikte wurden verschiedene moralisch gefärbte Selbst- und Fremdpositionierungen sichtbar, die auf die moralische Abgrenzung gegenüber bestimmten Personengruppen abzielten und die Zugehörigkeit zu anderen beanspruchten (vgl. Hirschauer 2014 und 2017).

So zeigte sich im ersten Interview, dass die gefühlte soziale Nähe zu anderen Therapieteilnehmern mit sehr deutlichen moralischen Abgrenzungen gegenüber diesen wie auch von früheren Weggefährt*innen einherging. Als Differenzierungslinien dienten hier vor allem die Arbeitswilligkeit, die alltägliche Lebensführung, die Fähigkeit zur Kontrolle des Drogenkonsums sowie Kriminalität. Die jeweiligen Selbstpositionierungen innerhalb dieser moralisch dimensionierten Kontinuen waren darauf gerichtet, die Anpassungsfähigkeit an gesellschaftliche Normalitätsvorstellungen zu bekunden. Dies wurde narrativ stellenweise durch die Reinszenierung von Situationen, in denen institutionelle Vertreter*innen die erreichte Position bestätigen und anerkennen, unterstrichen. Der Rückgriff auf biografische Ressourcen wie die frühe Arbeitsorientierung trotz intensiven Drogenkonsums vermittelte eine gewisse Kontinuität hinsichtlich der gegenwärtigen moralischen Position. Mit Bezug auf Kriminalität wurde die moralische Position zum einen durch eine Wendepunkterzählung gestärkt, die den schon vorhandenen Veränderungswillen betont. Zum anderen wurde durch die Positionierung 
als bereitwilliger Kooperationspartner der Ermittlungsbehörden der moralische Standpunkt als gesetzestreu bekräftigt.

Im Zusammenhang mit der Erzählung eines Wechsels der Fallzuständigkeit hin zu den Sozialbehörden wurden im zweiten Interview mehrfach Erfahrungen von stigmatisierenden $\gg$ Fehlkategorisierungen « (Hirschauer 2017, 40) geschildert. Hier wurde eine Kontextabhängigkeit des Anerkennungs- und Statuskonflikts besonders deutlich. Während der individuelle Lebensweg in der Drogentherapie Akzeptanz erfahren habe, sei er in den Kontakten mit den Sozialbehörden als Defizit markiert worden. Die Stärkung der eigenen moralischen Position durch Abgrenzung richtete sich dann gegen die Behördenvertreter*innen, wobei der Altersunterschied und die persönliche Lebenserfahrung als Differenzkategorien eingebracht wurden. Abgrenzungen gegenüber anderen Therapieteilnehmer*innen hingegen waren kaum erkennbar. Gesundheitliche Belastungen und eine gewisse Therapiemüdigkeit wurden als Gründe für einen wiedereinsetzenden Kokainkonsum angeführt, wodurch die Position als kontrollierter Konsument ins Wanken geriet. Widersprüche wurden dort sichtbar, wo einerseits die Eigenverantwortlichkeit für den Konsum und die Ablehnung von externalisierenden Erklärungen betont wurden, während die Unsicherheiten über die eigenen Konsumentscheidungen durch neurologische Diagnose- und Behandlungsverfahren ergründet werden sollten. Beides jedoch diente der moralischen Positionierung als angepasster Suchtkranker. In Hinblick auf Kriminalität geriet die moralische Positionierung als gesetzestreu ins Wanken, als die Vermeidung strafbarer Handlungen unter den belastenden Lebensumständen als herausfordernd beschrieben wurde.

Im dritten Interview hat sich vor dem Hintergrund der Erzählung über die erwünschte Beendigung der Fallzuständigkeit der Sozialbehörden und die Rückkehr in die Therapieeinrichtung eine Wiedererstarkung der ursprünglichen Positionierungsmuster gezeigt. Die Abgrenzungsaktivitäten entlang der moralischen Differenzierungslinien wurden erneut deutlich, insbesondere der Arbeitswille und die Kontrolle über den Drogenkonsum wurden narrativ herausgehoben. Hinsichtlich Kriminalität zeigte sich zum einen die Bekräftigung der Gesetzestreue durch die Bezugnahme auf die frühere Kooperationsgeschichte und das Zurückweisen von Angeboten des Wiedereinstiegs in den Drogenhandel. Zum anderen wurden Belastungen der Lebenssituation geschildert, denen der Entwurf einer Inhaftierung als entlastender Schonraum gegenübergestellt wurde.

Schließlich wurde im vierten Interview der gelungene Übertritt in den ersten Arbeitsmarkt beschrieben und eine Neuorientierung erkennbar. Die früheren Therapieteilnehmer*innen waren nicht mehr Ziel von Alterisierungen zur Sicherung der eigenen moralischen Position, sondern wurden in nostrifizierender Weise als zunehmend Schutzbedürftige entworfen. Differenzkonstruktionen richteten sich nun verstärkt auf verschiedene soziale Gruppen und deren Unterstützungswürdigkeit. Abgrenzungen wurden vor 
allem gegen Vertreter*innen des Großkapitals vorgenommen, deren Vergehen im Vergleich zu den eigenen moralisch abgewertet wurden. In Hinblick auf die Positionierung als kontrollierter Konsument zeigte sich dahingehend eine Verschiebung, dass durch die nachlassende Bedeutung der Therapieeinrichtung bestimmte Deutungsschemata, die erneuten Drogenkonsum als Scheitern markieren, zurückgewiesen wurden.

Ausgehend von diesen Ergebnissen lassen sich einige Erkenntnisse ableiten, die über die Spezifik des Einzelfalles hinausweisen. Der Forschungsbefund, dass die gefühlte soziale Nähe zu stigmatisierten Gruppen die Abgrenzungsbemühungen ihnen gegenüber erhöht, konnte auch anhand des analysierten Datenmaterials rekonstruiert werden (Goffman 1975; Copes 2016). Bisher wurde aber noch nicht untersucht, wie dynamisch dieses Wechselverhältnis im Zeitverlauf ist. Durch die Längsschnittbetrachtung konnte herausgearbeitet werden, dass moralische Positionierungen in spezifischer Weise kontextabhängig sind, sich also je nach Teilhabeerleben sowie Wahrnehmung des eigenen Status verändern. Hierbei scheinen institutionelle Kontexte eine bedeutsame Rolle zu spielen. Bemerkenswert ist, dass gerade als hilfreich und akzeptierend erfahrene Angebote mit einer moralischen Distanzierung von Personen in vergleichbaren Situationen einhergehen, während solche Maßnahmen, die als fremdbestimmend und kontrollierend erfahren werden, diese Abgrenzungsbemühungen abschwächen beziehungsweise auf die Institutionenvertreter*innen umleiten. Das Gefühl einer gelungenen Abkopplung von den Institutionen hingegen geht mit nostrifizierenden Positionierungen gegenüber den Personengruppen einher, die vorher als Bezugspunkt für moralische Abgrenzungen dienten.

\section{Anmerkungen}

1 Mit Desistance sind Prozesse des Abstandnehmens von kriminalisierbaren Handlungen gemeint. Statt danach zu fragen, wie eine kriminelle Karriere entsteht, ist zunehmend die Frage nach den Bedingungen und Mechanismen einer Beendigung derselben in den Fokus gerückt (Rieker et al. 2016).

2 Das Projekt »Wege aus der Straffälligkeit - Reintegrationsprozesse verurteilter Straftäter« unter der Leitung von Peter Rieker und Mitarbeit von Jakob Humm ist an der Universität Zürich angesiedelt und wird finanziell vom Schweizerischen Nationalfonds (SNF) gefördert.

3 In Zürich zum Beispiel entwickelte sich ab Mitte der 1980er Jahre eine offene Drogenszene, in der vor allem Heroin konsumiert und die 1995 geschlossen wurde. Da man die Lage mit repressiven Mitteln nicht in den Griff bekam, wurde die Drogenpolitik geändert und in der Folge vermehrt auch auf Maßnahmen der Schadensminimierung, Prävention und medizinischen Behandlung gesetzt (Uchtenhagen 2010).

4 Direkt wiedergegebene Rede von Dritten oder innere Monologe werden in den Interviewzitaten kursiv und in einfache Anführungszeichen gesetzt, um den Reinszenierungscharakter der Aussagen hervorzuheben. Die Transkriptionen wurden der hochdeutschen Sprache angepasst und stellenweise sprachlich geglättet. 


\section{Literatur}

Bereswill, Mechthild und Peter Rieker. 2008. "Selbstreflexion im Forschungsprozess und soziologische Theoriebildung". In Theoretische Empirie. Zur Relevanz qualitativer Forschung, hrsg. v. Herbert Kalthoff, Stefan Hirschauer und Gesa Lindemann, 399-431. Frankfurt a. M.: Suhrkamp.

Copes, Heith. 2016. »A Narrative Approach to Studying Symbolic Boundaries among Drug Users: A Qualitative Meta-Synthesis«. Crime Media Culture 12 (2): 193-213.

Goffman, Erving. 1975. Stigma. Über Techniken der Bewältigung beschädigter Identität. Frankfurt a. M.: Suhrkamp.

Groenemeyer, Axel. 2010. »Doing Social Problems - Doing Social Control. Mikroanalysen der Konstruktion sozialer Probleme in institutionellen Kontexten. Ein Forschungsprogramm«. In Doing Social Problems. Mikroanalysen der Konstruktion sozialer Probleme und sozialer Kontrolle in institutionellen Kontexten, hrsg. v. Axel Groenemeyer, 13-36. Wiesbaden: VS Verlag für Sozialwissenschaften.

Hanses, Andreas. 2010. „Gesundheit und Biographie - eine Gradwanderung zwischen Selbstoptimierung und Selbstsorge als gesellschaftliche Kritik». In Risiko Gesundheit. Über Risiken und Nebenwirkungen der Gesundheitsgesellschaft, hrsg. v. Bettina Paul und Henning Schmidt-Semisch, 89-103. Wiesbaden: VS Verlag für Sozialwissenschaften.

Hänninen, Vilma und Anja Koski-Jännes. 1999. »Narratives of Recovery from Addictive Behaviours«. Addiction 94 (12): 1837-48.

Hirschauer, Stefan. 2014. »Un/doing Differences. Die Kontingenz sozialer Zugehörigkeiten«. Zeitschrift für Soziologie 43 (3): 170-91.

Hirschauer, Stefan. 2017. »Humandifferenzierung. Modi und Grade sozialer Zugehörigkeit«. In Un/doing Differences. Praktiken der Humandifferenzierung, hrsg. v. Stefan Hirschauer, 29-54. Weilerswist: Velbrück.

Klein, Anna und Eva Groß. 2011. „Gerechte Abwertung? Gerechtigkeitsorientierungen und ihre Implikationen für schwache Gruppen«. In Gerechte Ausgrenzung? Wohlfahrtsproduktion und die neue Lust am Strafen, hrsg. v. Bernd Dollinger und Henning Schmidt-Semisch, 145-65. Wiesbaden: VS Verlag für Sozialwissenschaften.

Loseke, Donileen. 2007. »The Study of Identity as Cultural, Institutional, Organizational, and Personal Narratives: Theoretical and Empirical Integrations«. The Sociological Quarterly 48 (4): 661-88.

Lucius-Hoene, Gabriele und Arnulf Deppermann. 2004. „Narrative Identität und Positionierung«. Gesprächsforschung 5: 166-83.

Maruna, Shadd. 2001. Making Good. How Ex-Convicts Reform and Rebuild Their Lives. Washington, DC: American Psychological Association.

Maruna, Shadd und Heith Copes. 2005. "What have we learned from five decades of neutralization research?«. Crime and Justice 32: 221-320.

Maruna, Shadd, Thomas P. Lebel, Nick Mitchell und Michelle Naples. 2004. »Pygmalion in the Reintegration Process: Desistance from Crime Through the Looking Glass«. Psychology, Crime \& Law 10 (3): 271-81.

Maruna, Shadd und Kevin Roy. 2007. »Amputation or Reconstruction? Notes on the Concept of /Knifing Off and Desistance from Crime«. Journal of Contemporary Criminal Justice 23 (1): 104-24.

Miethe, Ingrid. 2014. »Neue Wege in der Biografieforschung: der Ansatz der theorieorientierten Fallrekonstruktion«. Zeitschrift für Qualitative Forschung 15 (1-2): 163-79.

Nadai, Eva. 2006. »Auf Bewährung. Arbeit und Aktivierung in Sozialhilfe und Arbeitslosenversicherung." Sozialer Sinn 7 (1): 61-77.

Nadai, Eva. 2009. „Das Problem der Bodensatzrosinen. Interinstitutionelle Kooperation und die forcierte Inklusion von Erwerbslosen«. Sozialer Sinn 10 (1): 55-71. 
Neckel, Sighard. 1991. Status und Scham. Zur symbolischen Reproduktion sozialer Ungleichheit. Frankfurt a. M. \& New York: Campus.

Pienaar, Kiran und Ella Dilkes-Frayne. 2017. »Telling Different Stories, Making New Realities: The Ontological Politics of `Addiction Biographies«. International Journal of Drug Policy 44: 145-54.

Reinarman, Craig. 2005. »Addiction as Accomplishment: The Discursive Construction of Disease Addiction Research \& Theory 13 (4): 307-20.

Rieker, Peter, Jakob Humm und Franz Zahradnik. 2016. „Einleitung: Desistance als konzeptioneller Rahmen für die Untersuchung von Reintegrationsprozessen«. Soziale Probleme 27 (2): 147-54.

Room, Robin. 2004. »The Cultural Framing of Addiction«. Janus Head 6 (2): 221-34.

Sandberg, Sveinung. 2016. »The Importance of Stories Untold: Life-Story, Event-Story and Trope«. Crime Media Culture 12 (2): 153-71.

Sandberg, Sveinung, Sébastien Tutenges und Heith Copes. 2015. „Stories of Violence: A Narrative Criminological Study of Ambiguity«. British Journal of Criminology 55: 1168-86.

Schmid, Otto, Thomas Müller, Gerhard A. Wiesbeck und Kenneth M. Dürstelr-MacFarland. 2009. »Felderhebung im Schweizer Drogenmilieu 2008«. Abhängigkeiten 3: 39-46.

Schmidt-Semisch, Henning. 2010. »Doing Addiction - Überlegungen zu Risiken und Nebenwirkungen des Suchtdiskurses«. In Risiko Gesundheit. Über Risiken und Nebenwirkungen der Gesundheitsgesellschaft, hrsg. v. Bettina Paul und Henning Schmidt-Semisch, 143-62. Wiesbaden: VS Verlag für Sozialwissenschaften.

Schmidt-Semisch, Henning und Jan Wehrheim. 2007. »Exkludierende Toleranz oder: Der halbierte Erfolg der sakzeptierenden Drogenarbeit«.. Widersprüche 27 (1): 73-91.

Strauss, Anselm und Juliet Corbin. 1996. Grounded Theory: Grundlagen qualitativer Sozialforschung. Weinheim: Psychologie Verlags Union.

Uchtenhagen, Ambros. 2010. »Heroin-Assisted Treatment in Switzerland: A Case Study in Policy Change«. Addiction 105: 29-37.

Weinberg, Darin. 2000. »Out Theres: The Ecology of Addiction in Drug Abuse Treatment Discourse«. Social Problems 47 (4): 606-21.

Witzel, Andreas. 2020. »Qualitative Längsschnittstudien«. In Handbuch Qualitative Forschung in der Psychologie, hrsg. v. Günter Mey und Katja Mruck, 19. Wiesbaden: VS Verlag für Sozialwissenschaften.

Zahradnik, Franz. 2018. Junge arbeitslose Männer in der Sanktionsspirale. Weinheim \& Basel: Beltz Juventa.

Zahradnik, Franz, Peter Rieker und Jakob Humm. 2019. »Die Bedeutung persönlicher Beziehungen im Kontext professioneller Hilfe und Kontrolle für die soziale Reintegration verurteilter Straftäter«. Bewährungshilfe 66 (3): 252-66.

\section{Der Autor}

Franz Zahradnik, Dr., wissenschaftlicher Projektmitarbeiter und Dozent an der Universität Zürich (UZH). Aktuelle Arbeitsschwerpunkte: Qualitative Längsschnittforschung, Positionierungsanalyse, Reintegration nach strafrechtlicher Verurteilung, subjektive Bedeutungszuschreibungen und Verarbeitungsweisen von Drogenkonsum.

Kontakt: Dr. Franz Zahradnik, Universität Zürich (UZH), Institut für Erziehungswissenschaft, Freiestrasse 36, 8032 Zürich, Schweiz; E-Mail: franz.zahradnik@ife.uzh.ch 